\title{
THE BOUNDED PROPER FORCING AXIOM AND WELL ORDERINGS OF THE REALS
}

\author{
Andrés Eduardo Caicedo And Boban VeličKović
}

\begin{abstract}
We show that the bounded proper forcing axiom BPFA implies that there is a well-ordering of $\mathcal{P}\left(\omega_{1}\right)$ which is $\Delta_{1}$ definable with parameter a subset of $\omega_{1}$. Our proof shows that if BPFA holds then any inner model of the universe of sets that correctly computes $\aleph_{2}$ and also satisfies BPFA must contain all subsets of $\omega_{1}$. We show as applications how to build minimal models of BPFA and that BPFA implies that the decision problem for the Härtig quantifier is not lightface projective.
\end{abstract}

\section{Introduction}

Forcing axioms are natural combinatorial statements which decide many of the questions left open by the usual axioms ZFC of set theory. The study of these axioms was started by Martin and Solovay [13] who introduced Martin's axiom MA as an abstraction of Solovay and Tennenbaum's approach to solving Suslin's problem [17], a question about uncountable trees. It was soon realized that MA together with the negation of the continuum hypothesis $\mathrm{CH}$ provides a rich structure theory for the reals. MA states a form of saturation of the universe of sets under possible sets added by forcing notions that satisfy the countable chain condition. As the method of forcing was further developed, generalizations of MA to larger classes of forcing posets were considered as well, most notably the proper forcing axiom PFA introduced by Baumgartner and Shelah (see, for example, Baumgartner's survey paper [3]) and Martin's maximum MM, the provably strongest forcing axiom, introduced by Foreman, Magidor and Shelah [7].

In recent years, bounded versions of traditional forcing axioms have received a considerable amount of attention as they have many of the same consequences, yet require much smaller large cardinal assumptions. These statements were first considered by Goldstern and Shelah in [8] who showed that the bounded proper forcing axiom BPFA is equiconsistent with a relatively modest large cardinal axiom, the existence of a $\Sigma_{1}$-reflecting cardinal. An appealing formulation of bounded forcing axioms as principles of generic absoluteness was provided by Bagaria [1]. Namely, suppose $\mathcal{K}$ is a class of forcing notions. The bounded forcing axiom $\operatorname{BFA}(\mathcal{K})$ is the statement asserting that for every $\mathcal{P} \in \mathcal{K}$,

$$
\left(H_{\aleph_{2}}, \in\right) \prec \Sigma_{1}\left(V^{\mathcal{P}}, \in\right) .
$$

Received by the editors July 28, 2005.

Key words and phrases. BPFA, MRP, definable well orderings, inner models, Härtig quantifier.

2000 Mathematics Subject Classification. Primary 03E05, 03E45, 03E47, 03E65; Secondary 03E40, $03 \mathrm{C} 80$. 
Here $H_{\aleph_{2}}$ denotes the collection of all sets whose transitive closure has size at most $\aleph_{1}$. Thus, BFA $(\mathcal{K})$ states that for every $\Sigma_{0}$ formula $\psi(x, a)$ with parameter $a \in H_{\aleph_{2}}$, if some forcing notion from $\mathcal{K}$ introduces a witness $x$ for $\psi(x, a)$, then such an $x$ already exists. For example, $\mathrm{MA}_{\aleph_{1}}$ is BFA(ccc), BPFA is BFA(Proper) and BMM is $\mathrm{BFA}(\mathcal{K})$ where $\mathcal{K}$ is the class of forcing notions that preserve stationary subsets of $\omega_{1}$.

One of the key questions left open by ZFC and resolved by forcing axioms stronger than $M_{\aleph_{1}}$ is the value of the continuum c. Thus, Foreman, Magidor and Shelah [7] showed that $M M$ implies that $\mathfrak{c}=\aleph_{2}$. The same conclusion was obtained from the weaker proper forcing axiom PFA by the second author [21] and Todorčević [4]. In [22] Woodin identified a statement $\psi_{\mathrm{AC}}$ which follows from both Woodin's $\mathcal{P}_{\text {max }^{-}}$ axiom $(*)$ and from $\mathrm{MM}$, and implies that $\mathfrak{c}=2^{\aleph_{1}}=\aleph_{2}$ and that there is a wellordering of the reals definable with parameters in $\left(H_{\aleph_{2}}, \in\right)$. Moreover, Woodin showed that BMM together with the existence of a measurable cardinal implies that the continuum is $\aleph_{2}$. The assumption of the existence of a measurable cardinal was later eliminated by Todorčević [20] who deduced these consequences of $\psi_{\mathrm{AC}}$ from a statement he called $\theta_{\mathrm{AC}}$ that he showed follows from BMM. Recently, Moore [14] introduced the mapping reflection principle MRP and deduced it from PFA. Although MRP does not follow from BPFA, Moore [14] used similar ideas to show that BPFA implies a certain statement $v_{\mathrm{AC}}$ which in turn implies that there is a well ordering of the reals, and in fact of $\mathcal{P}\left(\omega_{1}\right)$, of order type $\omega_{2}$ which is $\Delta_{2}$-definable in the structure $\left(H_{\aleph_{2}}, \in\right)$ with parameter a subset of $\omega_{1}$.

This paper continues this line of research. Our results were motivated by an attempt to extend the result of the second author [21] who showed that if MM holds and $M$ is an inner model such that $\omega_{2}^{M}=\omega_{2}$, then $\mathcal{P}\left(\omega_{1}\right) \subseteq M$. The interest in this study comes from the question, initially considered by Laver and Carlson (unpublished), of whether PFA can be preserved by some forcing notion in a nontrivial way. The best published result in this direction is the result of König and Yoshinobu [11, Theorem 6.1] who showed that PFA is preserved by $\omega_{2}$-closed forcing. The same holds for BPFA. In fact, BPFA is preserved by any proper forcing that does not add subsets of $\omega_{1}$. In this paper we use some of the ideas introduced by Moore [14] and design a robust coding of reals by triples of ordinals smaller than $\omega_{2}$. This allows us to show (Theorem 1) that if $M$ is an inner model, BPFA holds in both $M$ and $V$, and $\aleph_{2}^{M}=\aleph_{2}$, then $\mathcal{P}\left(\omega_{1}\right) \subseteq M$. This answers the question considered by Laver and Carlson negatively. Moreover, our coding allows us to show (Theorem 2) that BPFA implies the existence of a well ordering of the reals of optimal complexity, namely, $\Delta_{1}$ with parameter a subset of $\omega_{1}$. We then use these ideas to build a minimal model of BPFA and to show that under PFA the set of validities of the logic with the Härtig quantifier is not ordinal definable in $L(\mathbb{R})$. This last application was suggested to us by Jouko Väänänen in private communication.

Our notation is mostly standard. If $\kappa$ is an infinite cardinal, then $H_{\kappa}$ is the family of sets whose transitive closure has cardinality smaller than $\kappa$, i.e., $H_{\kappa}=\{x:|\operatorname{tc}(x)|<$ $\kappa\}$, where $\operatorname{tc}(\cdot)$ denotes transitive closure. We use $V^{\mathcal{P}}$ to denote the Boolean-valued extension of $V$ by the forcing notion $\mathcal{P}$, equivalently, and abusing language, $V^{\mathcal{P}}$ denotes any extension $V[G]$ where $G$ is $\mathcal{P}$-generic over $V$. A forcing $\mathcal{Q}$ is a factor of $\mathcal{P}$ iff $V^{\mathcal{Q}} \subseteq V^{\mathcal{P}}$, i.e., iff every $\mathcal{P}$-generic extension of $V$ contains an object $\mathcal{Q}$-generic over $V$. In this case, there is a forcing $\mathcal{B} \in V^{\mathcal{Q}}$ such that $\operatorname{ro}(\mathcal{P}) \cong \operatorname{ro}(\mathcal{Q}) * \dot{\mathcal{B}}$, and we 
write $\mathcal{B} \cong \operatorname{ro}(\mathcal{P}) / \operatorname{ro}(\mathcal{Q})$. We may on occasion abuse language and write $\mathcal{P}$ instead of $\operatorname{ro}(\mathcal{P})$, etc. Here, $\operatorname{ro}(\mathcal{P})$ denotes the regular open algebra of $\mathcal{P}$, i.e., the unique complete Boolean algebra into which $\mathcal{P}$ embeds densely. Suppose $V \subseteq M \subseteq V^{\mathcal{P}}$ where $M \models$ ZFC. Then there is a factor $\mathcal{Q}$ of $\mathcal{P}$ such that $M=V^{\mathcal{Q}}$ ([10, Lemma 15.43]). By a real we mean an element of the Cantor set $2^{\omega}$. If $X$ is a set and $(X, \in)$ satisfies extensionality, we let $\pi: X \rightarrow \bar{X}$ denote the Mostowski collapse of $X$, i.e., the unique isomorphism between $X$ and its transitive collapse. For all other notation, an introduction to set theory, and forcing see [10] to which we also refer for all concepts not defined explicitly in this paper.

This paper is organized as follows. In section 2 we reduce Theorem 1 and Theorem 2 to the verification of two facts. The proof of these two facts occupies sections 3 and 4 . Sections 5 and 6 contain applications of Theorem 1. In section 5 we build a minimal model of BPFA, solving a question of Sy Friedman. In section 6 we show that the set $V_{I}$ of validities of the logic with the Härtig quantifier is not lightface projective under BPFA and not even ordinal definable in $L(\mathbb{R})$ under PFA; this last result follows from a suggestion by Hugh Woodin that allowed us to improve our original result that PFA implies that $V_{I}$ is not definable in $L(\mathbb{R})$ without parameters.

The main results, Theorem 1 and Theorem 2, were obtained during the Fall of 2004, while the second author was visiting the Erwin Schrödinger Institute in Vienna. The results in section 5 and 6 were obtained during the Spring of 2005 while the first author was visiting the Université Denis-Diderot in Paris. We want to thank our respective hosts for their hospitality and generosity. We would also like to thank Jouko Väänänen for many useful conversations involving the content of this paper and Hugh Woodin for his suggestions on section 6.

\section{Coding reals}

The main results of this paper can be seen as consequences of Moore's mapping reflection principle MRP introduced in [14]. In [14, Theorem 1.1] it is shown that MRP is a consequence of PFA. Although BPFA is not sufficient to imply the full version of MRP we argue that it does imply the relevant consequences of MRP and thus we obtain the desired conclusions.

We start by describing a coding of reals by sets of ordinals reminiscent of Moore's $v_{\text {AC. }}$. We fix a $C$-sequence $\vec{C}=\left(C_{\xi}: \xi<\omega_{1}\right.$ a limit ordinal $)$, i.e., $C_{\xi}$ is an unbounded subset of $\xi$ of order type $\omega$ for all limit ordinals $\xi<\omega_{1}$. Given $x, y, z$ sets of natural numbers, define an equivalence relation $\sim_{x}$ on $\omega \backslash x$ by setting $n \sim_{x} m$ (for $n \leq m$ ) iff $[n, m] \cap x=\emptyset$. Thus the equivalence classes of $\sim_{x}$ are simply the intervals between the consecutive members of $x$. Let $\left(I_{k}\right)_{k \leq t}(t \leq \omega)$ be the natural enumeration of those equivalence classes which intersect both $y$ and $z$. In the cases that interest us $t$ will be finite, but, in general, $t=\omega$ is possible. Let the oscillation of $x, y, z$ be the function $o(x, y, z): t \rightarrow 2$ defined by letting for all $k<t$,

$$
o(x, y, z)(k)=0 \text { iff } \min \left(I_{k} \cap y\right) \leq \min \left(I_{k} \cap z\right) .
$$

Let $\omega_{1}<\beta<\gamma<\delta$ be fixed limit ordinals and suppose $N \subseteq M \subseteq \delta$ are countable sets of ordinals. Assume that $\left\{\omega_{1}, \beta, \gamma\right\} \subset N$, that $\sup (\xi \cap N)<\sup (\xi \cap M)$ and $\sup (\xi \cap M)$ is a limit ordinal, for every $\xi \in\left\{\omega_{1}, \beta, \gamma, \delta\right\}$. Then the pair $(N, M)$ codes a finite binary sequence as follows. Take the transitive collapse $\bar{M}$ of $M$ and let $\pi$ 
be the collapsing map. Let $\alpha_{M}=\pi\left(\omega_{1}\right), \beta_{M}=\pi(\beta), \gamma_{M}=\pi(\gamma), \delta_{M}=\bar{M}$, each of these is a countable limit ordinal. Let the height of $\alpha_{N}=\sup \left(\pi\left[\omega_{1} \cap N\right]\right)$ in $\alpha_{M}$ be the integer

$$
n=n(N, M)=\operatorname{card}\left(\alpha_{N} \cap C_{\alpha_{M}}\right) .
$$

Define three sets $x, y$ and $z$ of integers by

$$
x=\left\{\operatorname{card}\left(\pi(\xi) \cap C_{\beta_{M}}\right): \xi \in \beta \cap N\right\}
$$

and similarly for $y$ and $z$ with $\gamma$ and $\delta$, respectively, instead of $\beta$. Notice that $x, y, z$ are finite by our assumption on $N$ and $M$. Now, we look at the oscillation of $x \backslash n$, $y \backslash n$ and $z \backslash n$, which is a binary sequence, and if its length is at least $n$ then we let

$$
s_{\beta \gamma \delta}(N, M)=o(x \backslash n, y \backslash n, z \backslash n) \uparrow n .
$$

In all other cases we let $s_{\beta \gamma \delta}(N, M)=*$. We similarly write $s_{\beta \gamma \delta}(N, M) \uparrow l=*$ if $l>n(N, M)$.

Remark 1. Notice that there is a finite $T \subset N$ such that for any $S \subset N$, if $T \subset S$ then $s_{\beta \gamma \delta}(S, M)=s_{\beta \gamma \delta}(N, M)$. In effect, it suffices that $T$ contains $\left\{\omega_{1}, \beta, \gamma\right\}$, $\pi^{-1}\left[\alpha_{N} \cap C_{\alpha_{M}}\right]$, one point of $N$ for each interval in $\beta \cap M$ determined by $\pi^{-1}\left[C_{\beta_{M}}\right]$ that $N$ meets, and similarly for $\gamma$ and $\delta$.

Finally, we say that the triple $(\beta, \gamma, \delta)$ codes a real $r$ if there is a continuous increasing sequence $\left(N_{\xi}: \xi<\omega_{1}\right)$ of countable sets whose union is $\delta$ such that for every countable limit ordinal $\xi$ there is $\nu<\xi$ such that

$$
r=\bigcup_{\nu<\eta<\xi} s_{\beta \gamma \delta}\left(N_{\eta}, N_{\xi}\right) .
$$

We are going to show that BPFA implies the following two facts:

(1) Given ordinals $\omega_{1}<\beta<\gamma<\delta<\omega_{2}$ of cofinality $\omega_{1}$ there is an increasing continuous sequence $\left(N_{\xi}: \xi<\omega_{1}\right)$ of countable sets whose union is $\delta$ such that for every limit ordinal $\xi<\omega_{1}$ and every integer $n$ there is $\nu<\xi$ and $s_{\xi}^{n} \in\{0,1\}^{n} \cup\{*\}$ such that $s_{\beta \gamma \delta}\left(N_{\eta}, N_{\xi}\right) \backslash n=s_{\xi}^{n}$ for every $\eta$ such that $\nu<\eta<\xi$.

(2) For each real $r$ there are ordinals $\omega_{1}<\beta<\gamma<\delta<\omega_{2}$ of cofinality $\omega_{1}$ such that the triple $(\beta, \gamma, \delta)$ codes $r$.

Remark 2. Notice that in (1) we are not claiming that for a fixed $n$ the values of $s_{\xi}^{n}$ cohere as $\xi$ varies. However, by the way we have defined our coding it follows that for a fixed limit ordinal $\xi$ the values of $s_{\xi}^{n}$ (other than $*$ ) cohere as $n$ varies.

Before we proceed to the proof that BPFA implies (1) and (2) let us point out some consequences.

Theorem 1. Assume $M$ is an inner model, BPFA holds in both $M$ and $V$, and $\omega_{2}^{M}=\omega_{2}$. Then $\mathcal{P}\left(\omega_{1}\right) \subset M$. 
Proof: We claim that the theorem follows from (1) and (2). To see this, assume that $M$ is an inner model of $V$, that BPFA holds in both $M$ and $V$ and $\omega_{1}^{M}=\omega_{1}$. Fix a $C$-sequence in $M$ to carry out the codings just described. Suppose $\omega_{1}<\beta<$ $\gamma<\delta<\omega_{2}^{M}$ are ordinals of cofinality $\omega_{1}$ and the triple $(\beta, \gamma, \delta)$ codes in $V$ a real $r$. Let $\left(N_{\xi}: \xi<\omega_{1}\right)$ be a continuous increasing sequence of countable sets with union $\delta$ witnessing this. In $M$ there is a sequence $\left(P_{\xi}: \xi<\omega_{1}\right)$ witnessing $(1)$ for $(\beta, \gamma, \delta)$. There is a club $C \subseteq \omega_{1}$ in $V$ such that $N_{\xi}=P_{\xi}$, for every $\xi \in C$. Then it follows that for any $\xi$ which is a limit point of $C, r=\bigcup_{n} s_{\xi}^{n}$, as computed in $M$ relative to the sequence $\left(P_{\xi}: \xi<\omega_{1}\right)$. It follows that $r \in M$. If, moreover, $\omega_{2}=\omega_{2}^{M}$, then any real is coded in $V$ by some triple of ordinals less than $\omega_{2}^{M}$ and thus all reals are in $M$. But then $\mathcal{P}\left(\omega_{1}\right) \subseteq M$, since, given any $\omega_{1}$-sequence in $M$ of almost disjoint reals, BPFA, and in fact $\mathrm{MA}_{\aleph_{1}}$, allows us to code any subset of $\omega_{1}$ via this sequence and a real.

Recall that Moore showed in [14] that BPFA, in fact, the coding principle he named $v_{\text {AC }}$, implies that $2^{\aleph_{0}}=2^{\aleph_{1}}=\aleph_{2}$. Since BPFA implies $M_{\aleph_{1}}$, this also follows from our result.

Theorem 2. BPFA implies that there is a $\Delta_{1}$ well ordering of $\mathcal{P}\left(\omega_{1}\right)$ with parameter a subset of $\omega_{1}$. The length of the well ordering is $\omega_{2}$.

Proof: Fix as parameter a $C$-sequence $\vec{C}=\left(C_{\xi}: \xi<\omega_{1}\right.$ a limit ordinal). Let T be the theory "ZFC-Power set+MA $\mathrm{M}_{\aleph_{1}}+(1)+(2)+\forall x\left(|x| \leq \aleph_{1}\right)$ ". Notice that any transitive model $M$ of $\mathrm{T}$ which contains $\vec{C}$ is uniquely determined by $\operatorname{Ord} \cap M$. In effect, notice that since $\vec{C} \in M, M$ computes $\omega_{1}$ correctly. Suppose a real $r$ is coded by some triple $(\beta, \gamma, \delta)$ of ordinals in $M$. Then, arguing as in the proof of Theorem 1 , we see that $r \in M$. Notice that we are not claiming that $M$ knows that $(\beta, \gamma, \delta)$ codes $r$, just that $r \in M$. Since $M$ also satisfies (2) it follows that the reals in $M$ are precisely the reals coded by some triple of ordinals which belong to $M$. Since $M A_{\aleph_{1}}$ holds in $M$ it follows that $\mathcal{P}\left(\omega_{1}\right)^{M}$ is completely determined as well. Namely, from $\vec{C}$ we can define a canonical $\omega_{1}$-sequence $\vec{r}$ of almost disjoint reals, and we can use the standard almost disjoint coding to code a subset of $\omega_{1}$ by the sequence $\vec{r}$ and a real. Now, for an ordinal $\theta<\omega_{2}$, let $M_{\theta}$ be the unique transitive model $M$ of $\mathrm{T}$ containing $\vec{C}$ such that $\operatorname{Ord} \cap M=\theta$, if it exists; otherwise let $M_{\theta}=\emptyset$. Notice that the function $\theta \mapsto M_{\theta}$ is $\Delta_{1}$ in the parameter $\vec{C}$.

Now, let $<_{*}$ be the antilexicographic ordering on the class $[\mathrm{Ord}]^{3}$ of increasing triples of ordinals. For a real $r$ let $\theta_{r}$ be the least $\theta$ such that $r \in M_{\theta}$ and let $\left(\beta_{r}, \gamma_{r}, \delta_{r}\right)$ be the $<_{*}$-least triple of ordinals smaller than $\theta_{r}$ such that $M_{\theta_{r}} \models\left(\beta_{r}, \gamma_{r}, \delta_{r}\right)$ codes $r$. Finally, let $r \triangleleft s$ iff either $\theta_{r}<\theta_{s}$ or $\theta_{r}=\theta_{s}$ and $\left(\beta_{r}, \gamma_{r}, \delta_{r}\right)<_{*}\left(\beta_{s}, \gamma_{s}, \delta_{s}\right)$.

We can now define a well ordering $\prec$ of $\mathcal{P}\left(\omega_{1}\right)$ as follows. For $a$ and $b$ subsets of $\omega_{1}$, we define $a \prec b$ iff the $\triangleleft$-least real coding $a$ from the sequence $\vec{r}$ of almost disjoint reals defined from $\vec{C}$ is $\triangleleft$-smaller than the $\triangleleft$-least real coding $b$. By an argument similar to the above, $\prec$ is also $\Delta_{1}$ in the parameter $\vec{C}$.

As an additional bonus, notice that the set of possible parameters in the definition of the well ordering, i.e., the set of $C$-sequences, is $\Delta_{0}$ in the parameter $\omega_{1}$. 
Remark 3. The readers familiar with [14] may find in order a few words contrasting our Theorems 1 and 2 with the results in [14], specifically Moore's result that BPFA implies that there is a well-ordering of $\mathcal{P}\left(\omega_{1}\right)$ definable over $\left(H_{\aleph_{2}}, \in, \mathrm{NS}_{\omega_{1}}\right)$ from a $C$ sequence. In [14] the principle $v_{\mathrm{AC}}$ is introduced and shown to follow from BPFA. From $v_{\text {AC }}$ it follows that each equivalence class $[S] \in \mathcal{P}\left(\omega_{1}\right) / \mathrm{NS}_{\omega_{1}}$ is coded by an ordinal $\delta<\omega_{2}$ of cofinality $\omega_{1}$, where $\mathrm{NS}_{\omega_{1}}$ denotes the nonstationary ideal on $\omega_{1}$. This corresponds to the statement we numbered (2) above. The statement corresponding to (1), namely that any such $\delta$ codes some equivalence class $[S]$, is also a consequence of BPFA as can be easily seen from the arguments in [14] even though it is not explicitly mentioned there, alternately one can argue as in section 3 below. However, it seems that these versions of (1) and (2) do not suffice to prove Theorem 1. The standard way in which $\mathcal{P}(\omega)$ is coded once we have $v_{\mathrm{AC}}$ (or Woodin's $\psi_{\mathrm{AC}}$, or Todorčević's $\theta_{\mathrm{AC}}$ ) consists of fixing a partition $\vec{S}=\left(S_{n}: n<\omega\right)$ of $\omega_{1}$ into stationary sets and assigning to a real $r \in 2^{\omega}$ the ordinal corresponding to $\left[S_{r}\right]$, where $S_{r}=\bigcup_{r(n)=1} S_{n}$.

Suppose that $M$ is an inner model of $V$, that $\omega_{2}^{M}=\omega_{2}$, and that BPFA holds in both $M$ and $V$. Suppose that a partition $\vec{S} \in M$ as above is given such that for each $n, S_{n}$ is still stationary in $V$. Such partitions actually exist, as Larson shows in [12]. In $V$ let $r$ be a real and let $\delta$ be an ordinal coding $r$ relative to the partition $\vec{S}$. In $M, \delta$ codes the equivalence class of some set $A$. It follows that in $V$, the symmetric difference of $A$ and $S_{r}$ is nonstationary. However, this is not enough to conclude that $r \in M$. Namely, for any $n$ such that $r(n)=0, S_{n} \backslash A$ is stationary in $M$ since it is stationary in $V$, but, for some $n$ such that $r(n)=1$, it may be a priori that $S_{n} \backslash A$ is a stationary set in $M$ whose stationarity is destroyed in $V$. This means that in $M$, we may not be able to recover $r$ from $A$. And even if $r \in M$, it may be that $A \backslash S_{r}$ is stationary in $M$ but not in $V$, so we may fail to identify correctly in $M$ the ordinals that code $r$ in $V$.

These considerations lead us to look for a coding of reals by triple of ordinals similar to the one given by $v_{\mathrm{AC}}$ but not so explicitly dependent on $\mathrm{NS}_{\omega_{1}}$, and this ultimately resulted in the versions of (1) and (2) mentioned above.

Now consider the complexity of the well-ordering obtained in Theorem 2 and the one obtained in [14]. Using $v_{\mathrm{AC}}$ and fixing a partition $\vec{S}$ of $\omega_{1}$ into stationary sets, for each real $r$ let $\delta_{r}$ be the least ordinal which codes the equivalence class of $S_{r}$. We say $r<s$ iff $\delta_{r}<\delta_{s}$. To say that $r<s$ one needs to express that there is an ordinal $\delta$ which codes $S_{r}$ (this part is $\Sigma_{1}$ ) and such that for all ordinals $\mu<\delta$ the set coded by $\mu$ is not equal to $S_{s}$ modulo $\mathrm{NS}_{\omega_{1}}$. The point is that this second part adds another quantifier, since expressing that the symmetric difference of the set coded by $\mu$ and $S_{s}$ is stationary is a $\Pi_{1}$ statement.

Remark 4. It is open whether even MM implies that there is a definable well ordering of the reals without parameters.

Corollary 1. Assume BPFA holds and $\omega_{1}=\omega_{1}^{L}$. Then there is a projective wellordering of the reals.

Proof: The argument is very similar to the one in [5], so we only sketch the main points. Just notice that in $L$ there is a $C$-sequence $\vec{C}$ that is $\Delta_{2}^{1}$ in the codes. By Shoenfield absoluteness, $\vec{C}$ is still $\Delta_{2}^{1}$ in $V$ and, since $\omega_{1}=\omega_{1}^{L}$, it is still a $C$-sequence. 
From $\vec{C}$, we can define a well-ordering of $\mathbb{R}$ by looking at ordinals in $S_{\omega_{1}}^{\omega_{2}}$, but they can be coded as subsets of $\omega_{1}$, which can be coded by reals using almost disjoint forcing, for this we can use a $\Delta_{2}^{1}$ sequence in $L$ of almost disjoint reals as the $\omega_{1}$-sequence relative to which the almost disjoint coding takes place. As shown in [5], these codings are projective.

Remark 5. The well ordering obtained in Corollary 1 is in general not optimal. Friedman has shown the consistency of BPFA together with a $\Sigma_{3}^{1}$ well ordering of the reals by class forcing techniques, see [6].

\section{Open partitions}

The purpose of this section is to show that BPFA implies (1). We start by recalling the relevant definitions from [14].

Definition 1 ([14, Definition 2.1]). Let $\theta$ be a regular cardinal, let $X$ be uncountable, and let $M \prec H_{\theta}$ be countable such that $[X]^{\omega} \in M$. A subset $\Sigma$ of $[X]^{\omega}$ is $M$-stationary iff for all $E \in M$ such that $E \subseteq[X]^{\omega}$ is club, $\Sigma \cap E \cap M \neq \emptyset$.

Recall that the Ellentuck topology on $[X]^{\omega}$ is obtained by declaring a set open iff it is the union of sets of the form

$$
[x, N]=\left\{Y \in[X]^{\omega}: x \subseteq Y \subseteq N\right\}
$$

where $N \in[X]^{\omega}$ and $x \subseteq N$ is finite. When we say 'open' in this paper we refer to this topology.

Definition 2 ([14, Definition 2.3]). A set mapping $\Sigma$ is open stationary iff there is an uncountable set $X=X_{\Sigma}$ and a regular cardinal $\theta=\theta_{\Sigma}$ such that $[X]^{\omega} \in H_{\theta}$, $\operatorname{dom}(\Sigma)$ is a club in $\left[H_{\theta}\right]^{\omega}$ and $\Sigma(M) \subseteq[X]^{\omega}$ is open and $M$-stationary, for every $M$ in the domain of $\Sigma$.

Recall the Mapping Reflection Principle (MRP) introduced in [14].

If $\Sigma$ is an open stationary set mapping whose domain is a club, there is a continuous $\in$-chain $\vec{N}=\left(N_{\xi}: \xi<\omega_{1}\right)$ of elements in the domain of $\Sigma$ such that for all limit ordinals $\xi<\omega_{1}$ there is $\nu<\xi$ such that $N_{\eta} \cap X_{\Sigma} \in \Sigma\left(N_{\xi}\right)$ for all $\eta$ such that $\nu<\eta<\xi$.

If $\left(N_{\xi}: \xi<\omega_{1}\right)$ satisfies the conclusion of MRP for $\Sigma$ then it is said to be a reflecting sequence for $\Sigma$. It is shown in [14] that MRP is a consequence of PFA. In fact, what is proved in [14] is that for a given open stationary set mapping $\Sigma$ there is a proper forcing notion which introduces a reflecting sequence for $\Sigma$.

Lemma 1. Assume MRP. Suppose that for each $\alpha<\omega_{1}$ we have a partition $\alpha=$ $K_{\alpha}^{0} \cup K_{\alpha}^{1}$ into clopen sets in the standard order topology on $\alpha$. Then there a club $C \subset \omega_{1}$ such that for every limit point $\xi$ of $C$ there is $i \in 2$ such that $C \backslash K_{\xi}^{i}$ is bounded in $\xi$. Moreover, this conclusion follows from BPFA.

Proof: Let $X=\omega_{1}$ and let $\theta$ be a sufficiently large regular cardinal. Notice that $\omega_{1}$ is a closed subset of $\left[\omega_{1}\right]^{\omega}$ in the Ellentuck topology and the relativization of this topology to $\omega_{1}$ is just the usual order topology on $\omega_{1}$. 
Let $M \prec H_{\theta}$ be countable and let $\alpha=M \cap \omega_{1}$. We claim that one of the $K_{\alpha}^{i}$ $(i \in 2)$ is $M$-stationary. Otherwise, there are $E_{0}, E_{1} \in M$ club subsets of $\omega_{1}$ such that $E_{i} \cap K_{\alpha}^{i}=\emptyset(i \in 2)$ and therefore $E_{0} \cap E_{1}=\emptyset$ by elementarity. Define $\Sigma$ by letting $\Sigma(M)=\left[\omega_{1}\right]^{\omega} \backslash K_{\alpha}^{i}$ for the $i$ such that $K_{\alpha}^{1-i}$ is $M$-stationary. Now, applying MRP we obtain a reflecting sequence $\left(N_{\xi}: \xi<\omega_{1}\right)$ for $\Sigma$. Let $C=\left\{N_{\xi} \cap \omega_{1}: \xi<\omega_{1}\right\}$. It is clear that $C$ is as required. To see that the conclusion follows from BPFA notice that the existence of $C$ as stated is a $\Sigma_{1}$ statement with parameter $\left(K_{\alpha}^{i}: \alpha<\omega_{1}, i \in 2\right)$. Since any particular instance of MRP can be forced by a proper forcing it follows that BPFA implies the conclusion of the lemma.

Lemma 2. Assume BPFA. Then (1) holds.

Proof: Fix a triple of ordinals $\omega_{1}<\beta<\gamma<\delta<\omega_{2}$, each of cofinality $\omega_{1}$. We first find an increasing continuous sequence $\left(N_{\xi}: \xi<\omega_{1}\right)$ of countable sets with union $\delta$. By thinning out the sequence, if necessary, we may assume that $\left\{\omega_{1}, \beta, \gamma\right\} \subseteq N_{0}$, that $\sup \left(\rho \cap N_{\xi}\right)$ is a limit ordinal and $\sup \left(\rho \cap N_{\eta}\right)<\sup \left(\rho \cap N_{\xi}\right)$, for every $\eta<\xi<\omega_{1}$ and $\rho \in\left\{\omega_{1}, \beta, \gamma, \delta\right\}$. Fix an integer $n$ and define, for each countable limit ordinal $\xi$, a partition of $\xi$ into $2^{n}+1$ disjoint sets, $\xi=\bigcup_{s \in 2^{n} \cup\{*\}} K_{\xi}^{s}$ by

$$
\eta \in K_{\xi}^{s} \quad \text { iff } \quad s_{\beta \gamma \delta}\left(N_{\eta}, N_{\xi}\right) \uparrow n=s .
$$

By Remark 1 each piece of the partition is open in the order topology on $\omega_{1}$ and since the pieces are disjoint and cover $\xi$ they are also closed. By applying Lemma 1 we find a closed unbounded subset $C_{n}$ of $\omega_{1}$ and for each limit point $\xi$ of $C_{n}$, an element $s_{\xi}^{n}$ of $2^{n} \cup\{*\}$ such that there is $\nu<\xi$ such that $s_{\beta \gamma \delta}\left(N_{\eta}, N_{\xi}\right)\left\lceil n=s_{\xi}^{n}\right.$ for every $\eta \in C_{n}$ such that $\nu<\eta<\xi$. Finally, let $C=\bigcap_{n} C_{n}$. Then the sequence $\left(N_{\xi}: \xi \in C\right)$ witnesses (1) for the triple $(\beta, \gamma, \delta)$.

\section{Playing games}

Now we show that BPFA implies (2). As before we first show that MRP implies (2) and then argue that BPFA suffices. In order to show that the appropriate set mapping is open stationary we shall use a game argument reminiscent of [21, Lemma 3.7]. For a given algebra $F:\left[\omega_{4}\right]^{<\omega} \rightarrow \omega_{4}$, we define a sequence of games $\mathcal{G}_{\nu}^{F}$ indexed by countable ordinals $\nu$. These are $\omega$-length perfect information games between two players I and II playing alternately. We show that these games are determined and that player II has a winning strategy for almost all of them. Playing against these strategies allows us to build models where any desired oscillation pattern occurs. This implies that for every real $r$ a certain map $\Sigma^{r}$ to be defined below is open stationary, from which (2) follows immediately in the presence of MRP. We then argue as in Lemma 1 to show that BPFA is sufficient to imply (2). The game $\mathcal{G}_{\nu}^{F}$ is defined as follows:

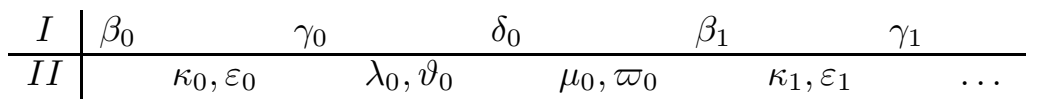

As indicated in the diagram, player I plays, alternately, ordinals $\beta_{i}, \gamma_{i}$ or $\delta_{i}$ such that $\left(\beta_{i}\right)_{i},\left(\gamma_{i}\right)_{i}$ and $\left(\delta_{i}\right)_{i}$ are increasing sequences of ordinals in $\omega_{2}, \omega_{3}$ and $\omega_{4}$ respectively.

Player II responds in turn by playing, alternately,

- ordinals $\kappa_{i}, \varepsilon_{i}$ such that $\beta_{i} \leq \kappa_{i} \leq \varepsilon_{i}<\aleph_{2}$. 
- ordinals $\lambda_{i}, \vartheta_{i}$ such that $\gamma_{i} \leq \lambda_{i} \leq \vartheta_{i}<\aleph_{3}$.

- ordinals $\mu_{i}, \varpi_{i}$ such that $\delta_{i} \leq \mu_{i} \leq \varpi_{i}<\aleph_{4}$.

We also require, although this is not really essential, that

- $\varepsilon_{i}<\beta_{i+1}, \vartheta_{i}<\gamma_{i+1}$ and $\varpi_{i}<\delta_{i+1}$, for all $i<\omega$.

The first player to violate these rules loses. Otherwise, let

$$
X=\operatorname{cl}_{F}\left(\left\{\kappa_{n}, \lambda_{n}, \mu_{n}: n<\omega\right\} \cup \nu\right),
$$

where $\mathrm{cl}_{F}$ denotes closure under $F$. Then we say that player II wins iff

- $X \cap \omega_{1}=\nu$,

- $X \cap\left[\beta_{n}, \beta_{n+1}\right) \subseteq\left[\beta_{n}, \varepsilon_{n}\right)$ for all $n$,

- $X \cap\left[\gamma_{n}, \gamma_{n+1}\right) \subseteq\left[\gamma_{n}, \vartheta_{n}\right)$ for all $n$, and

- $X \cap\left[\delta_{n}, \delta_{n+1}\right) \subseteq\left[\delta_{n}, \varpi_{n}\right)$ for all $n$.

It follows that if player I wins a particular run of $\mathcal{G}_{\nu}^{F}$ then this happens at some finite stage. By the Gale-Stewart theorem $\mathcal{G}_{\nu}^{F}$ is determined, for all $\nu$. We now show that player II wins $\mathcal{G}_{\nu}^{F}$ for club many $\nu$. Let

$$
A_{F}=\left\{\nu<\omega_{1}: \text { I has a winning strategy in } \mathcal{G}_{\nu}^{F}\right\} .
$$

Lemma 3. $A_{F}$ is nonstationary.

Proof: This is similar to [21, Lemma 3.7]. Assume towards contradiction that $A_{F}$ is stationary and fix, for each $\nu \in A_{F}$, a winning strategy $\sigma_{\nu}$ for $\mathrm{I}$ in $\mathcal{G}_{\nu}^{F}$. Since $A_{F}$ has cardinality $\aleph_{1}$ there is a strategy $\sigma$ which dominates all the $\sigma_{\nu}$. Namely, at any given position when it is player I's turn, $\sigma$ looks at what the strategies $\sigma_{\nu}$ would play, for $\nu \in A_{F}$, and plays the supremum of these ordinals. Then clearly $\sigma$ is a winning strategy for I in $\mathcal{G}_{\nu}^{F}$ for all $\nu \in A_{F}$. Moreover, we can assume that at every stage when it is player I's turn to play an ordinal $\gamma_{n}<\omega_{3}$ the move played by $\sigma$ does not depend on the previous move of player II. This is possible since there are only $\aleph_{2}$ possible previous moves of player II, so we look at what $\sigma$ would play against each of them and play the supremum of these ordinals. Similarly, we may assume that at every stage when it is player I's turn to play an ordinal $\delta_{n}<\omega_{4}$, the move played by $\sigma$ does not depend on the two previous moves of player II. We call this property of $\sigma$ stability.

We now describe a play of II against $\sigma$. Fix a sufficiently large regular cardinal $\theta$ and build an increasing continuous chain

$$
P_{0} \prec P_{1} \prec \cdots \prec P_{\xi} \prec \cdots \prec H_{\theta}, \quad \xi<\omega_{1} \cdot \omega
$$

of elementary submodels of $H_{\theta}$ of size $\aleph_{1}$ such that $F, A_{F}, \sigma \in P_{0}$. Let $N_{n}=P_{\omega_{1} \cdot n}$ and $N=\bigcup_{n} N_{n}$. Notice that, for all $n$, the ordinals $\zeta_{n}=\sup \left(N_{n} \cap \omega_{2}\right), \eta_{n}=\sup \left(N_{n} \cap \omega_{3}\right)$ and $\theta_{n}=\sup \left(N_{n} \cap \omega_{4}\right)$ all have cofinality $\omega_{1}$ and $N_{n}$ contains as subsets $\omega_{1}$-club subsets of each of these ordinals that belong as elements to $N_{n+1}$. Since we assumed that $A_{F}$ is stationary we can find a countable $M \prec N$ such that $\left\{\zeta_{n}, \eta_{n}, \theta_{n}\right\}_{n} \subseteq M$, $F, \sigma, A_{F} \in M$, all the club sets mentioned above belong to $M$, and $\alpha=M \cap \omega_{1} \in A_{F}$. We describe a run of $\mathcal{G}_{\alpha}^{F}$ in which player I plays following $\sigma$. We consider the game as consisting of rounds of three moves of each player, one in each of $\omega_{2}, \omega_{3}$ and $\omega_{4}$.

Suppose that we are at the start of the $n$-th round, the current position is $p_{n-1}=$ $\left\langle\beta_{0},\left(\kappa_{0}, \varepsilon_{0}\right), \ldots, \delta_{n-1},\left(\mu_{n-1}, \varpi_{n-1}\right)\right\rangle$, and we have arranged that $p_{n-1} \in N_{n}$. Player I then plays $\beta_{n}=\sigma\left(p_{n-1}\right)$. Since $\sigma, p_{n-1} \in N_{n}$ it follows that $\beta_{n} \in N_{n}$. Player 
II picks an ordinal $\kappa_{n} \in M \cap N_{n+1}$ such that $\zeta_{n} \leq \kappa_{n}<\omega_{2}$. Let $\varepsilon_{n}=\sup (M \cap$ $\left.N_{n+1} \cap \omega_{2}\right)$. Since $M$ is countable and $\zeta_{n+1}=\sup \left(N_{n+1} \cap \omega_{2}\right)$ has cofinality $\omega_{1}$ it follows that $\varepsilon_{n}<\zeta_{n+1}$. In fact, since $N_{n+1}$ contains a club in $\zeta_{n+1}$ that belongs to $M$, it follows that $\varepsilon_{n} \in N_{n+1}$. Player II then plays $\left(\kappa_{n}, \varepsilon_{n}\right)$. Player I responds by playing $\sigma\left(p_{n-1} \widehat{\langle}\left\langle\beta_{n},\left(\kappa_{n}, \varepsilon_{n}\right)\right\rangle\right)=\gamma_{n}$. By elementarity of $N_{n}$ and stability of $\sigma$ it follows that $\gamma_{n} \in N_{n}$. Player II then picks some $\lambda_{n} \in M \cap N_{n+1}$ such that $\eta_{n} \leq \lambda_{n}<\omega_{3}$. Let $\vartheta_{n}=\sup \left(M \cap N_{n+1} \cap \omega_{3}\right)$. By the same argument as above, $\vartheta_{n}<\eta_{n+1}$ and $\vartheta_{n} \in N_{n+1}$. Player II then plays $\left(\lambda_{n}, \vartheta_{n}\right)$. Player I responds by playing $\delta_{n}=\sigma\left(p_{n-1} \widehat{\uparrow}\left\langle\beta_{n},\left(\kappa_{n}, \varepsilon_{n}\right), \gamma_{n},\left(\lambda_{n}, \vartheta_{n}\right)\right\rangle\right)$. Again, by elementarity of $N_{n}$ and stability of $\sigma$ it follows that $\delta_{n} \in N_{n}$. Player II picks some ordinal $\mu_{n} \in M \cap$ $N_{n+1}$ such that $\theta_{n} \leq \mu_{n}<\omega_{4}$. Let $\varpi_{n}=\sup \left(M \cap N_{n+1} \cap \omega_{4}\right)$. Again, we have that $\varpi_{n}<\theta_{n+1}$ and, in fact, $\varpi_{n} \in N_{n+1}$. Player II then plays $\left(\mu_{n}, \varpi_{n}\right)$. Let $p_{n}=p_{n-1} \hat{\langle}\left\langle\beta_{n},\left(\kappa_{n}, \varepsilon_{n}\right), \gamma_{n},\left(\lambda_{n}, \vartheta_{n}\right), \delta_{n},\left(\mu_{n}, \varpi_{n}\right)\right\rangle$. It follows that $p_{n} \in N_{n+1}$. This completes the inductive construction of the play. Let

$$
X=\operatorname{cl}_{F}\left(\left\{\kappa_{i}, \lambda_{i}, \mu_{i}: i<\omega\right\} \cup \alpha\right) .
$$

Notice that $X \subseteq M$. This implies that $X \cap \omega_{1}=\alpha$. Also since $\beta_{n+1} \in N_{n+1}$ and $\varepsilon_{n}=\sup \left(M \cap N_{n+1} \cap \omega_{2}\right)$ it follows that $X \cap\left[\beta_{n}, \beta_{n+1}\right) \subseteq\left[\beta_{n}, \varepsilon_{n}\right)$, for all $n$. Similarly, we have that $X \cap\left[\gamma_{n}, \gamma_{n+1}\right) \subseteq\left[\gamma_{n}, \vartheta_{n}\right)$, and $X \cap\left[\delta_{n}, \delta_{n+1}\right) \subseteq\left[\delta_{n}, \varpi_{n}\right)$, for all $n$. Thus, player II has won this run of $\mathcal{G}_{\alpha}^{F}$, a contradiction.

Lemma 4. Assume BPFA. Then for every real $r$ there is a triple of ordinals less than $\omega_{2}$ coding r, i.e., (2) holds.

Proof: Fix a real $r$. As before we will first derive the desired conclusion from MRP and then argue that it also follows from BPFA. We start by defining an open stationary set mapping $\Sigma^{r}$. Fix a sufficiently large regular cardinal $\theta$. For a countable elementary submodel $M$ of $H_{\theta}$ define

$$
\Sigma^{r}(M)=\left\{N \in\left[M \cap \omega_{4}\right]^{\omega}: s_{\omega_{2} \omega_{3} \omega_{4}}\left(N, M \cap \omega_{4}\right) \text { is an initial segment of } r\right\} .
$$

By Remark 1, $\Sigma^{r}(M)$ is open in the Ellentuck topology on $\left[\omega_{4}\right]^{\omega}$. We will show that it is also $M$-stationary. Assume this for a moment and let us see how to finish the proof.

Assume first MRP and let $\left(M_{\xi}: \xi<\omega_{1}\right)$ be a reflecting sequence for $\Sigma^{r}$. Let $M=\bigcup_{\xi<\omega_{1}} M_{\xi}$, let $\bar{M}$ be the transitive collapse of $M$ and let $\pi$ be the collapsing map. Let $\beta=\pi\left(\omega_{2}\right), \gamma=\pi\left(\omega_{3}\right)$ and $\delta=\pi\left(\omega_{4}\right)$. Then $\omega_{1}<\beta<\gamma<\delta<\omega_{2}$ and $\operatorname{cof}(\beta)=\operatorname{cof}(\gamma)=\operatorname{cof}(\delta)=\omega_{1}$. Let $N_{\xi}=\pi\left(M_{\xi} \cap \omega_{4}\right)$. Note that $s_{\beta \gamma \delta}\left(N_{\eta}, N_{\xi}\right)=$ $s_{\omega_{2} \omega_{3} \omega_{4}}\left(M_{\eta} \cap \omega_{4}, M_{\xi} \cap \omega_{4}\right)$ for every $\eta<\xi<\omega_{1}$. Moreover, for $\xi$ a limit ordinal, $N_{\xi}=\bigcup_{\eta<\xi} N_{\eta}$ and therefore $\lim _{\eta<\xi} n\left(N_{\eta}, N_{\xi}\right)=\omega$. It follows that for some $\nu<\xi$,

$$
r=\bigcup_{\nu<\eta<\xi} s_{\beta \gamma \delta}\left(N_{\eta}, N_{\xi}\right)
$$

Therefore the triple $(\beta, \gamma, \delta)$ codes $r$ as desired. To see that the same conclusion follows from BPFA note that there is a proper forcing notion adding a reflecting sequence for $\Sigma^{r}$ and the existence of a triple coding $r$ is a $\Sigma_{1}$ statement in parameter the $C$-sequence used for the coding and the real $r$. Therefore the existence of such a triple of ordinals follows from BPFA. To complete the proof of Lemma 4 it remains to show the following. 
Lemma 5. $\Sigma^{r}$ is a stationary set mapping.

Proof: Let $M$ be a countable elementary submodel of $H_{\theta}$. We have to show that $\Sigma^{r}(M)$ is $M$-stationary. We work in $M$ and fix an algebra $F$ on $\omega_{4}$. By Lemma 3 we can find a club $C$ in $\omega_{1}$ and for every $\nu \in C$ a winning strategy $\sigma_{\nu}$ for player II in $\mathcal{G}_{\nu}^{F}$. We show that we can fix an ordinal $\nu \in C \cap M$ and play finitely many moves of the game $\mathcal{G}_{\nu}^{F}$ with ordinals inside $M$ such that if $T$ is the finite set of relevant ordinals played by player II and $X=\operatorname{cl}_{F}(T \cup \nu)$ then $s_{\omega_{2} \omega_{3} \omega_{4}}\left(X, M \cap \omega_{4}\right)$ is an initial segment of $r$. We will never leave $M$, even though we refer to objects which do not belong to $M$ to assist us with our choices.

Let $\bar{M}$ be the transitive collapse of $M$ and let $\pi$ be the collapsing map. Let $\alpha_{M}=$ $\pi\left(\omega_{1}\right), \beta_{M}=\pi\left(\omega_{2}\right), \gamma_{M}=\pi\left(\omega_{3}\right) \delta_{M}=\pi\left(\omega_{4}\right)$. Recall that we have fixed a $C$-sequence $\left(C_{\xi}: \xi<\omega_{1}\right)$. If $\rho \in M \cap \omega_{4}$, the height of $\rho$ in $\omega_{i}, \operatorname{ht}_{\omega_{i}}(\rho)$, is $\operatorname{card}\left(\pi(\rho) \cap C_{\pi\left(\omega_{i}\right)}\right)$, for $i=1, \ldots, 4$. Of course, the height of $\rho$ in $\omega_{i}$ is known only outside of $M$, however we only need to refer to the heights of finitely many ordinals.

Now, look at what the strategies $\sigma_{\nu}$ do at the first step when player I plays 0 . For each $\nu \in C, \sigma_{\nu}(\langle 0\rangle)=\left(\kappa_{0}^{\nu}, \varepsilon_{0}^{\nu}\right)$ is a pair of ordinals below $\omega_{2}$ with $\kappa_{0}^{\nu} \leq \varepsilon_{0}^{\nu}$. Let $\varepsilon_{0} \in \omega_{2} \cap M$ be the supremum of all the $\varepsilon_{0}^{\nu}$. Similarly, let $\left(\lambda_{0}^{\nu}, \vartheta_{0}^{\nu}\right)=\sigma_{\nu}\left(\left\langle 0, \sigma_{\nu}(\langle 0\rangle), 0\right\rangle\right)$, and let $\vartheta_{0} \in M \cap \omega_{3}$ be the supremum of all the $\vartheta_{0}^{\nu}$. Define $\varpi_{0}$ analogously. Stepping out of $M$ for a moment, look at the height of $\varepsilon_{0}$ in $\omega_{2}$ and similarly with $\vartheta_{0}$ and $\varpi_{0}$. Let $k$ be the largest of these integers. We now choose $\nu \in C \cap M$ be such that its height in $\omega_{1}$, say $n$, is bigger than $k$. We are going to play the game $\mathcal{G}_{\nu}^{F}$ and player II is going to use his winning strategy $\sigma_{\nu}$.

The first round of the play, consisting of three moves for each player, is a bit special. In it, player I simply plays 0 three times and player II responds according to $\sigma_{\nu}$. Let us say that the three moves of player II in this round are $\left(\kappa_{0}, \varepsilon_{0}\right),\left(\lambda_{0}, \vartheta_{0}\right)$ and $\left(\mu_{0}, \varpi_{0}\right)$. By the choice of $\nu$ we have that the height of $\varepsilon_{0}$ in $\omega_{2}$, the height of $\vartheta_{0}$ in $\omega_{3}$ and the height of $\varpi_{0}$ in $\omega_{4}$ are all smaller than $n$.

Suppose we have played rounds $0, \ldots, i-1$ and the moves of player II in the last round are $\left(\kappa_{i-1}, \varepsilon_{i-1}\right),\left(\lambda_{i-1}, \vartheta_{i-1}\right)$ and $\left(\mu_{i-1}, \varpi_{i-1}\right)$. In the next round we have to code $r(i-1)$. Player I first chooses $\beta_{i}$ such that

$$
\operatorname{ht}_{\omega_{2}}\left(\beta_{i}\right)>\max \left\{\operatorname{ht}_{\omega_{3}}\left(\vartheta_{i-1}\right), \text { ht }_{\omega_{4}}\left(\varpi_{i-1}\right), n\right\} .
$$

Player II responds using $\sigma_{\nu}$ and plays, say, $\left(\kappa_{i}, \varepsilon_{i}\right)$. We have $\beta_{i} \leq \kappa_{i} \leq \varepsilon_{i}$. Then there are two cases.

Case 1. $r(i-1)=0$. Player I then chooses $\gamma_{i}$ such that ht $\omega_{\omega_{3}}\left(\gamma_{i}\right)>\mathrm{ht}_{\omega_{2}}\left(\varepsilon_{i}\right)$. Player II follows $\sigma_{\nu}$ and plays, say, $\left(\lambda_{i}, \vartheta_{i}\right)$. Of course, we have that $\gamma_{i} \leq \lambda_{i} \leq \vartheta_{i}$. Player I then plays $\delta_{i}$ such that $\mathrm{ht}_{\omega_{4}}\left(\delta_{i}\right)>\mathrm{ht}_{\omega_{3}}\left(\vartheta_{i}\right)$ and player II, using $\sigma_{\nu}$, plays, say, $\left(\mu_{i}, \varpi_{i}\right)$. Of course, we have $\delta_{i} \leq \mu_{i} \leq \varpi_{i}$. Therefore, the height in $\omega_{2}$ of any point of the interval $\left[\beta_{i}, \varepsilon_{i}\right)$ is smaller than the height in $\omega_{3}$ of any point of the interval $\left[\gamma_{i}, \vartheta_{i}\right)$ which in turn is smaller than the height in $\omega_{4}$ of any point of the interval $\left[\delta_{i}, \varpi_{i}\right)$.

Case 2. $r(i-1)=1$. First fix an ordinal $\xi \in \omega_{4} \cap M$ such that ht $\omega_{\omega_{4}}(\xi)>$ ht $_{\omega_{2}}\left(\varepsilon_{i}\right)$. For each move, say, $\gamma<\omega_{3}$ of player I, suppose the strategy $\sigma_{\nu}$ responds by $(\lambda(\gamma), \vartheta(\gamma))$. Now, suppose that if player I then plays $\xi$ the strategy $\sigma_{\nu}$ responds by $(\mu(\gamma), \varpi(\gamma))$. Let $\varpi=\sup \left\{\varpi(\gamma): \gamma<\omega_{3}\right\}$. Player I then plays some $\gamma_{i} \in \omega_{3} \cap M$ such that 
$\operatorname{ht}_{\omega_{3}}\left(\gamma_{i}\right)>$ ht $_{\omega_{4}}(\varpi)$. Player II then responds according to $\sigma_{\nu}$ and player I then plays $\xi$ and player II responds by using $\sigma_{\nu}$ and plays, say, $\left(\mu_{i}, \varpi_{i}\right)$. In this way we ensure that $\mathrm{ht}_{\omega_{3}}\left(\gamma_{i}\right)>\mathrm{ht}_{\omega_{4}}\left(\varpi_{i}\right)$. Therefore, the height in $\omega_{2}$ of any point of the interval $\left[\beta_{i}, \varepsilon_{i}\right)$ is smaller than the height in $\omega_{4}$ of any point of the interval $\left[\delta_{i}, \varpi_{i}\right)$ which is smaller than the height in $\omega_{3}$ of any point of the interval $\left[\gamma_{i}, \vartheta_{i}\right)$.

We play this game for $n+1$ rounds and code in this way the first $n$ digits of $r$. Finally, let $X=\operatorname{cl}_{F}\left(\left\{\kappa_{i}, \lambda_{i}, \mu_{i}: i \leq n+1\right\} \cup \nu\right)$. Since $\sigma_{\nu}$ is a winning strategy for player II in $\mathcal{G}_{\alpha}^{F}$, by our construction it follows that $s_{\omega_{2} \omega_{3} \omega_{4}}\left(X, M \cap \omega_{4}\right)=r\lceil n$. Thus, $X \in \Sigma^{r}(M)$. Since $F$ is an arbitrary algebra on $\omega_{4}$ and $X$ is closed under $F$ it follows that $\Sigma^{r}(M)$ is $M$-stationary. This completes the proof of Lemma 5 and Lemma 4.

\section{A minimal model of BPFA}

Let $\phi$ be a sentence in the language of set theory. Let us say that a model $M$ of ZFC $+\phi$ is minimal for $\phi$ iff for any inner model $N \subseteq M$ with the same ordinals, if $N$ is also a model of ZFC $+\phi$ then $N=M$.

Recall ([8]) that an uncountable regular cardinal $\kappa$ is $\Sigma_{1}$-reflecting (or $H_{\kappa}$-reflecting) iff for every $a \in H_{\kappa}$ and every formula $\varphi(x)$, if there is a regular cardinal $\theta$ such that $H_{\theta} \models \varphi(a)$ then there is a $\theta^{\prime}<\kappa$ such that $a \in H_{\theta^{\prime}}$ and $H_{\theta^{\prime}} \models \varphi(a)$.

In [8, Theorem 2.11], Goldstern and Shelah show that if $\kappa$ is $\Sigma_{1}$-reflecting, then there is a countable support iteration of length $\kappa$ of proper forcing notions of size smaller than $\kappa$ that forces BPFA. For future reference, we sketch the construction. We define a countable support iteration of proper forcing notions of size less than $\kappa$, $\left\langle\mathcal{P}_{\alpha} ; \dot{\mathcal{Q}}_{\alpha}: \alpha<\kappa\right\rangle$, and let $\mathcal{P}_{\kappa}$ be their direct limit. At stage $\alpha$ we have some $\Sigma_{1}$ formula and a parameter which is a subset of $\omega_{1}$, and ask if there is a proper poset which adds a solution to this formula. If the answer is yes then, since $\kappa$ is $\Sigma_{1}$-reflecting, there is such a poset of size less than $\kappa$ and we choose one as $\mathcal{Q}_{\alpha}$. We do this using some standard bookkeeping device so every subset of $\omega_{1}$ appearing along the iteration and every $\Sigma_{1}$ formula are considered at cofinally many stages. We will refer to this $\mathcal{P}_{\kappa}$ as the standard iteration for BPFA. Conversely, by [8, Theorem 4.1], if BPFA holds then $\aleph_{2}$ is $\Sigma_{1}$-reflecting in $L$.

Let $\kappa$ be the smallest $\Sigma_{1}$-reflecting cardinal in $L$ and let $G$ be generic for the standard iteration $\mathcal{P}_{\kappa}$ for BPFA. We argue in this section that there is an inner model $M$ of $L[G]$ which is a minimal model for BPFA. This answers a question of Sy Friedman (private communication).

Theorem 3. Suppose that $\kappa$ is a $\Sigma_{1}$-reflecting cardinal in $V$ and that $\mathcal{P}_{\kappa}$ is the standard iteration for BPFA. Let $M=V\left(\mathcal{P}\left(\omega_{1}\right)^{V^{\mathcal{P}_{\kappa}}}\right)$. Then $M \models$ BPFA.

Proof: Recall that if $N$ is an inner model of the universe $V$ and $X$ is a set, then $N(X)=\bigcup_{\alpha} L\left(N_{\alpha} \cup \mathrm{tc}(\{X\})\right)$, where $N_{\alpha}=N \cap V_{\alpha}$ and the union is over all the ordinals. This is a model of ZF. In particular, $M$ is a model of ZF. It is in fact a model of Choice, by the argument of Theorem 2. Let $\mathcal{Q}$ be the complete subalgebra of $\operatorname{ro}\left(\mathcal{P}_{\kappa}\right)$ such that $V^{\mathcal{Q}}=M$. Then $\mathcal{Q}$ is proper. Let $a \subseteq \omega_{1}$ (so $a \in M$ ) and let $\varphi$ be a $\Sigma_{1}$ formula, say $\varphi$ is $\exists x \psi(x, a)$, where $\psi$ is $\Sigma_{0}$. Since $\mathcal{P}_{\kappa}$ is $\kappa$-cc, there is an $\alpha<\kappa$ such that $a \in V^{\mathcal{P}_{\alpha}}$ and $a$ has size $\aleph_{1}$ there. The formula $\varphi$ and the parameter $a$ are considered at some stage $\beta>\alpha$ of the iteration. Notice that $V^{\mathcal{P}_{\beta}} \subseteq V^{\mathcal{Q}}$. Since 
the tail of the iteration $\mathcal{P}_{\kappa} / \mathcal{P}_{\beta}$ is proper in $V^{\mathcal{P}_{\beta}}$ and $\mathcal{Q} / \mathcal{P}_{\beta}$ is a factor of it, $\mathcal{Q} / \mathcal{P}_{\beta}$ is proper in $V^{\mathcal{P}_{\beta}}$, as well. It follows that if there is a proper forcing $\mathcal{R} \in M$ adding to $M$ a witness to $\varphi(a)$ then, since $\mathcal{Q} / \mathcal{P}_{\beta} * \dot{\mathcal{R}}$ is proper, at stage $\beta$ there was a proper forcing adding a witness to $\varphi(a)$. Therefore, $\mathcal{Q}_{\beta}$ was chosen to add such a witness. It follows that $M$ satisfies BPFA.

Corollary 2. In $L$, let $\kappa$ be the smallest $\Sigma_{1}$-reflecting cardinal and let $\mathcal{P}_{\kappa}$ be the standard iteration for BPFA of length $\kappa$. Let $M=L\left(\mathcal{P}\left(\omega_{1}\right)\right)^{L^{\mathcal{P}_{\kappa}}}$. Then $M$ is a minimal model of BPFA.

Proof: By Theorem 3, $M$ is a model of ZFC + BPFA. Assume that $N$ is an inner model of $M$ and that $N \models$ BPFA. By minimality of $\kappa, \omega_{2}^{N}=\kappa=\omega_{2}^{M}$, so Theorem 1 applies, and $\mathcal{P}\left(\omega_{1}\right)^{M} \subset N$, so $N=M$.

Remark 6. Let $\kappa$ be $\Sigma_{1}$-reflecting over $V$ and suppose that $\mathcal{P}_{\kappa}$ is given by the standard iteration for BPFA. Let $G$ be $\mathcal{P}_{\kappa}$-generic over $V$. It would be interesting to know whether $V\left(\mathcal{P}\left(\omega_{1}\right)^{V[G]}\right)$ is actually different from $V[G]$. Notice that the forcing extending $V\left(\mathcal{P}\left(\omega_{1}\right)^{V[G]}\right)$ to $V[G]$ is $\omega$-distributive and $\omega_{2}$-cc. If the ground model is $L$, it is even $\omega_{1}$-distributive. The problem is that, even though the initial parts $G_{\alpha}=G \cap \mathcal{P}_{\alpha}$ of the generic $G$ belong to $V\left(\mathcal{P}\left(\omega_{1}\right)\right)$, they may not be the only $\mathcal{P}_{\alpha^{-}}$ generic filters in $V\left(\mathcal{P}\left(\omega_{1}\right)\right)$. The question is whether we can glue them together to get the whole generic $G$, i.e., we have to choose generic filters for initial segments of the iteration which cohere.

Working over $L$, or any other definable generically invariant class with definable well-orderings, like, for example, the Dodd-Jensen core model $K_{D J}$, one can modify slightly the forcing $\mathcal{P}_{\kappa}$ so that it has in addition a certain amount of rigidity: if in $V$ there are two different $\mathcal{P}_{\kappa}$-generic filters over $L$ then $\omega_{1}^{L}$ is collapsed. We use the following fact that we proved in section 4 . Given a $C$-sequence and any real $r$, there is a proper forcing $\mathcal{P}_{r}$ of size $\aleph_{4}^{\aleph_{0}}$ collapsing $\aleph_{4}$ to $\aleph_{1}$ such that in $V^{\mathcal{P}_{r}}$ the triple $\left(\omega_{2}^{V}, \omega_{3}^{V}, \omega_{4}^{V}\right)$ codes $r$.

Let $\kappa$ be a reflecting cardinal. We define $\left\langle\mathcal{P}_{\alpha} ; \dot{\mathcal{Q}}_{\alpha}: \alpha<\kappa\right\rangle$ and let $\mathcal{P}_{\kappa}$ be their countable support limit. At odd stages $\alpha$ we proceed as before, i.e., we choose the forcing $\mathcal{Q}_{\alpha}$ of least $\mathcal{P}_{\alpha}$-name in the sense of the natural well ordering of $L$. If $\alpha$ is even, $\mathcal{Q}_{\alpha}$ does the following. Let $\lambda$ be $\left|\mathcal{P}_{\alpha}\right|^{L}$, so $\lambda<\kappa$. We first collapse $\lambda$ to $\omega_{1}$ with countable conditions. Now, the whole generic up to this point, including the collapse, can be coded by a subset $a_{\alpha}$ of $\omega_{1}$, and then using almost disjoint coding relative to a fixed definable sequence of almost disjoint reals in $L$, we code $a_{\alpha}$ by a real, say $r=r_{\alpha}$. We know the values of $\aleph_{2}, \aleph_{3}$ and $\aleph_{4}$ of this model, since they are just the $L$-successors of $\lambda$ (actually we know all the cardinals, but we only need these ones). For simplicity let us call them $\beta<\gamma<\delta$. We then force with $\mathcal{P}_{r}$. It is easy to see that $\mathcal{P}_{\kappa}$ thus obtained forces BPFA.

Notice that at any even stage $\alpha$, the real coded by $(\beta, \gamma, \delta)$ cannot change in any outer model with the same $\omega_{1}$, as being a club in $\omega_{1}$ is upwards absolute between models with the same $\omega_{1}$, and this real is seen to be $r$ in the extension by $\mathcal{P}_{\kappa}$. In fact, this coding will be done anyway at some triple (not necessarily $(\beta, \gamma, \delta)$ ) when we force BPFA in the usual way, but the point is that we code the whole generic up to stage $\alpha$ by these three ordinals which moreover we can identify in the ground model. 
Now, in the end, the generic $G$ will actually be definable without parameters, as it can be reconstructed inductively from its initial segments, which can be recovered using the reals $r_{\alpha}$. Moreover, $G$ will be unique in any outer model which has the same $\omega_{1}$ as $L$. It follows that $V=L\left(\mathcal{P}\left(\omega_{1}\right)\right)$ holds in $L[G]$.

The canonicity in the choice of $\mathcal{Q}_{\alpha}$ for odd stages $\alpha$ and a careful choice of bookkeeping gives us that if $\lambda<\kappa$ is a smaller reflecting cardinal in $L$, then the $\lambda^{\text {th }}$ stage of the iteration $\mathcal{P}_{\lambda}$ also forces BPFA over $L$. In particular, $L[G]$ will not be a minimal model for BPFA unless $\kappa$ is the $L$-least reflecting cardinal. This leads naturally to the question of whether if $M$ is a minimal model for BPFA, then $\kappa=\omega_{2}^{M}$ is the least reflecting cardinal in $L$. The argument above does not answer this question, as there may be of course a poset $\mathcal{Q} \in L$ forcing BPFA or even BPFA $+V=L\left(\mathcal{P}\left(\omega_{1}\right)\right)$ but such that no $\mathcal{P}_{\kappa}$ as above is a factor of $\mathcal{Q}$.

\section{The complexity of the logic with the Härtig quantifier}

In this section we present as an application of the main result, an observation suggested by Jouko Väänänen on the complexity of the set of validities of the Härtig quantifier.

Definition 3. The logic $\mathcal{L}(I)$ is obtained by augmenting first-order logic with a binary quantifier $I$ - the Härtig quantifier. Formulas in $\mathcal{L}(I)$ are defined recursively as in first-order logic, with the additional clause:

$$
\begin{gathered}
\text { If } \varphi(x, \vec{z}) \text { and } \psi(y, \vec{z}) \text { are formulas in } \mathcal{L}(I), \text { then so is } \\
\operatorname{Ixy}(\phi(x, \vec{z}), \psi(y, \vec{z})),
\end{gathered}
$$

where $x$ and $y$ are considered bound variables.

The semantics of $\mathcal{L}(I)$ is defined recursively as in first-order logic, with the additional clause:

$$
\begin{aligned}
& \mathcal{M}=(M, \ldots) \models \operatorname{Ixy}(\phi(x, \vec{a}), \psi(y, \vec{a})) \text { iff } \\
& \qquad|\{b \in M: \mathcal{M} \models \phi(b, \vec{a})\}|=|\{c \in M: \mathcal{M} \models \psi(c, \vec{a})\}| .
\end{aligned}
$$

In a straightforward fashion, we can assign Gödel numbers to the formulas in $\mathcal{L}(I)$. Let $V_{I}$ be the set of validities of the logic $\mathcal{L}(I)$, i.e., the set of (Gödel numbers for) sentences in $\mathcal{L}(I)$ valid in all models. We refer the reader to [9] for an introduction to the Härtig quantifier and for the results we mention in the argument below. This proof is closely modeled on the argument given in [9] that $V_{I}$ is neither $\Sigma_{2}^{1}$ nor $\Pi_{2}^{1}$.

Corollary 3. Suppose that BPFA holds. Then $V_{I}$ is not lightface projective.

Proof: We show that in fact $V_{I}$ is not first-order definable (without parameters) over $N=L_{\aleph_{2}^{V}}\left(\mathcal{P}\left(\omega_{1}\right)\right)$ so, in particular, it is not lightface projective.

Let $n$ be a sufficiently large integer and denote by ZFC ${ }_{n}$ the finitely axiomatizable theory that results from restricting the replacement schema of ZFC to $\Sigma_{n}$-formulas. The integer $n$ is chosen so Theorem 1 is provable in $\mathrm{ZFC}_{n}$ and also the argument below goes through.

Let $\varphi$ be a first order sentence without parameters in the language of set theory. We find an $\mathcal{L}(I)$-formula $\psi_{\varphi}$ such that $N \models \varphi$ iff $\psi_{\varphi} \in V_{I}$. Since $\varphi$ is arbitrary, it follows that $V_{I}$ cannot be first order definable over $N$. 
Our sentence $\psi_{\varphi}$ has the form $\phi \rightarrow \theta_{\varphi}$, where $\theta_{\varphi}$ is a first order sentence (in the language of set theory) expressing the statement $N \models \varphi$. $\phi$ will be such that its models are precisely the well-founded models of ZFC ${ }_{n}+$ BPFA whose transitive collapse correctly computes $\aleph_{2}$. Suppose $M$ is such a model. Then, by Theorem 1 , it correctly computes $N$, and the equivalence follows.

All that remains is to exhibit $\phi$. In [9] it is explained how to produce, for any finite extension $T$ of a sufficiently strong fragment of ZFC, an $\mathcal{L}(I)$-sentence $\phi_{T}$ such that a structure $(M, E)$ is a well-founded model of $T$ iff it admits an expansion to a model of $\phi_{T}$. Let $\phi_{\mathbb{N}}(x), \phi_{\omega_{1}}(x)$ and $\phi_{\omega_{2}}(x)$ be the first order formulas stating, respectively, that $x$ is a natural number, that $x$ is a countable ordinal, and that $x$ is an ordinal below $\omega_{2}$. Our $\phi$ is the conjunction of $\phi_{\mathrm{ZFC}_{n}+\operatorname{BPFA}}$ and $\neg \operatorname{Ixy}\left(\phi_{\mathbb{N}}(x), \phi_{\omega_{1}}(y)\right) \wedge$ $\neg \operatorname{Ixy}\left(\phi_{\omega_{1}}(x), \phi_{\omega_{2}}(y)\right)$, and it is easy to see that $\phi$ is as wanted. This completes the proof. Notice that the map $\varphi \mapsto \psi_{\varphi}$ is recursive.

Remark 7. It is easy to see by condensation and a similar argument, that $V=L$ implies that $V_{I}$ is not $\Sigma_{n}^{m}$ for any $n$ and any $m<\omega$ (or even $\Sigma_{n}^{\alpha}$ for any $n$ and any $\alpha<\omega_{1}^{C K}$ ). This has also been observed by Väänänen (private communication).

The following argument incorporates a suggestion of Woodin that strengthens and simplifies our original argument. Assume PFA holds. By the covering lemma and the fact that PFA implies $\neg \square_{\kappa}$ for all $\kappa \geq \omega_{1}$, (see [19]), it follows that $X^{\sharp}$ exists for all sets $X$. In particular, $\mathbb{R}^{\sharp}$ exists. By Theorem 1 , if $N$ is an inner model of BPFA ( $N$ may be a set) that correctly computes $\omega_{2}$, then $H_{\aleph_{2}} \subseteq N$. If $N$ is also a model of PFA then there is an $X \in N$ such that $N \models X=\mathbb{R}^{\sharp}$, but then $X$ really is $\mathbb{R}^{\sharp}$ (see [16] for the definition and basic properties of $\mathbb{R}^{\sharp}$, from which this follows). Since $\mathbb{R}^{\sharp}$ codes the theory of $L(\mathbb{R})$, this easily implies as above that the theory of $L(\mathbb{R})$ without parameters is recursive in $V_{I}$ and therefore $V_{I}$ is not definable in $L(\mathbb{R})$ without parameters.

But something stronger in fact holds. By Steel [18], PFA implies that $\mathrm{AD}^{L(\mathbb{R})}$ holds. Solovay [16] has shown that $A D^{L(\mathbb{R})}$ and the existence of $\mathbb{R}^{\sharp}$ imply that there is a real which is ordinal definable in $L\left(\mathbb{R}^{\sharp}\right)$ but not ordinal definable in $L(\mathbb{R})$. Since the least such real in the natural well-ordering of $\operatorname{HOD}^{L\left(\mathbb{R}^{\sharp}\right)}$ is obviously recursive in $V_{I}$ by the arguments above, it follows that $V_{I}$ itself cannot be ordinal definable in $L(\mathbb{R})$.

Corollary 4. Assume PFA. Then $V_{I}$ is not ordinal definable in $L(\mathbb{R})$.

\section{References}

[1] J. Bagaria, Bounded forcing axioms as principles of generic absoluteness, Arch. Math. Logic 39 (2000), no. 6, 393-401.

[2] J. Baumgartner, Iterated forcing, Iterated forcing. Surveys in set theory, 1-59, London Math. Soc. Lecture Note Ser., 87, Cambridge Univ. Press, Cambridge, 1983.

[3] _ Applications of the proper forcing axiom, Handbook of set-theoretic topology, NorthHolland (1984) 913-959.

[4] M. Bekkali, Topics in set theory, Springer (1991).

[5] A. Caicedo, Projective well-orderings and bounded forcing axioms, J. Symbolic Logic 70 (2005), no. $2,557-572$.

[6] A. Caicedo, S. Friedman. Projective well-orderings of the reals: A survey, in preparation. 
[7] M. Foreman, M. Magidor, and S. Shelah, Martin's maximum, saturated ideals, and nonregular ultrafilters. I, Ann. of Math. (2) 127 (1988), no. 1, 1-47.

[8] M. Goldstern and S. Shelah, The bounded proper forcing axiom, J. Symbolic Logic 60 (1995), no. $1,58-73$.

[9] H. Herre, M. Krynicky, A. Pinus, and J. Väänänen, The Härtig quantifier: A survey, J. Symbolic Logic 56 (1991), no. 4, 1153-1183.

[10] T. Jech, Set theory, the third millenium edition, Springer (2003).

[11] B. König and Y. Yoshinobu, Fragments of Martin's Maximum in generic extensions, MLQ Math. Log. Q. 50 (2004), no. 3, 297-302.

[12] P. Larson, The nonstationary ideal in the $\mathcal{P}_{\max }$ extension, preprint.

[13] D. Martin and R. Solovay, Internal Cohen extensions, Ann. Math. Logic 2 (1970), no. 2, 143178.

[14] J. Moore, Set mapping reflection, J. Math. Log. 5 (2005), no. 1, 87-98.

[15] S. Shelah, Proper and improper forcing, Springer (1998).

[16] R. Solovay, The independence of DC from AD, in Cabal Seminar 76-77 (Proceedings of the UCLA-Caltech logic seminar, 1976-77), A. Kechris, Y. Moschovakis, eds., Springer (1978) 171183.

[17] R. Solovay and S. Tennenbaum, Iterated Cohen extensions and Souslin's problem, Ann. Math. 94 (1971), no. 2, 201-245

[18] J. Steel, PFA implies $\mathrm{AD}^{L(\mathbb{R})}$, preprint.

[19] S. Todorčević, A note on the proper forcing axiom, in Axiomatic set theory (Boulder, Colorado, 1983), J. Baumgartner, D. Martin, eds., Amer. Math. Soc. (1984), 209-218.

[20] _ Generic absoluteness and the continuum, Math. Res. Lett. 9 (2002) 1-7.

[21] B. Veličković, Forcing axioms and stationary sets, Adv. Math. 94 (1992), no. 2, 256-284.

[22] H. Woodin, The axiom of determinacy, forcing axioms, and the nonstationary ideal, Walter de Gruyter (1999).

Department of Mathematics, Mail code 253-37, California Institute of Technology, Pasadena, CA 91125

E-mail address: caicedo@caltech.edu

Equipe de Logique Mathématique, UFR de Mathématiques (CASe 7012), Université DenisDiderot Paris 7, 2 place Jussieu, 75251 Paris Cedex 05, France

E-mail address: boban@logique.jussieu.fr 